\title{
Denoising of MRI images using fast NLM
}

\author{
Vandana Hanchate ${ }^{1}$, Kalyani Joshi ${ }^{2}$ \\ ${ }^{1}$ College Of Engineering, Savitribai Phule Pune University, India \\ ${ }^{2}$ PES's Modern College of Engineering, Savitribai Phule Pune University, India
}

\begin{tabular}{l}
\hline \hline Article Info \\
\hline Article history: \\
Received Aug 1, 2019 \\
Revised Sep 19, 2019 \\
Accepted Oct 6, 2019 \\
\hline Keywords: \\
MSE \\
NLM \\
PFOM \\
PSNR \\
SSIM
\end{tabular}

\begin{abstract}
Denoising of image is a very crucial step which should retain fine details but should remove noise. Making the difference between noise and actual edge related data is very difficult. NLM filter helps to make a differentiation between image data and noise data. Its weight function decides the weightage of the neighboring pixel depending upon the similarity with the pixel to process. It helps to retain the edges and avoid it from smoothening. This paper discusses the implementation of NLM filter using hardware platform Spartan 6. After implementaion of this on FPGA, not only denoise the image but preseve edges and there is a tremendous saving in time compared to its matlab implementation. Denoised image performance is calculated using various objective metrics such as MSE, PSNR, SSIM, PFOM etc. FPGA implementation shows clearly the advntages over its matlab implementation.
\end{abstract}

Copyright $\odot 2020$ Institute of Advanced Engineering and Science. All rights reserved.

\section{Corresponding Author:}

Vandana Hanchate,

Department of E\&TC, College of Engineering,

Savitribai Phule Pune University, India.

Email: vnjpune@gmail.com

\section{INTRODUCTION}

Medical imaging became an integral part of disease diagnosis in present days. Various medical modalities are available depending upon the application. The use of this modalities is to produce the clear imaging of anatomical structure within the body helping us to diagnose the malfunctioning of the body. The popular modalities available are X-rays, Computed Tomography, Nuclear imaging, Magnetic Resonance Imaging and Ultrasound to diagnose the various diseases. However, the common problem associated with all theses modality while imaging is noise. Due to this noise, the intensity values in the image will vary from its original i.e. noise produces unwanted changes in the medical images leading to misdiagonosis. Noise is not introduced only while capturing the image but it also introduces while transmission, compression etc. Various factors are responsible for introduction of this noise in the resulting image. Every modality is affected by a different type of noise such as Nuclear imaging and X-rays are affected by quantum noise, Magnetic resonance imaging is affected by Rician noise, ultrasound imaging by speckle noise etc. This ultimately decreases the image contrast and misleads in diagnostic phase. So denoising is very important step to remove the noise from medical images [1].

Magnetic Resonance Imaging is a useful imaging technique to provide precise detailed images of internal organs and tissues in the human body. Nuclear Magneic resonance (NMR) is the main working principle of MRI system. It uses the concept of there is change in the nuclear spin when a specific proton or nuclei is kept in the magnetic field. By capturing this spin and by mapping it to spatial location MRI image is formed. Human body has $80 \%$ of water content and water is formation of hydrogen and oxygen. When hydrogen atoms are placed in strong magnetic field and excited by the resonant magnetic excitation pulse, there is change in the spin and when the excitation is removed then atom will return back to its original spin while radaiating the waves. These waves are imaged to demonstrate the abnormal changes into human tissues [2]. 
MRI is having a limitation of limited aquisation time based on the patient's comfort. This leads to low SNR of the MR images. The qualities of the magnetic resonance images are generally degraded with several artifact and noises that is adequately modeled as Rician noise. In the Rician noise model, the observed MR image intensities are non-linear function of the true image intensities and it is signal dependent noise and this adds bias to the medical images denoised by conventional denoising methods. So, a method which will remove noise from the image whereas will retain the structural fine details is a very important step in MR image denoising. While designing the algorithm for the denoising of MRI the main objective in front of many researches to provide images with both high SNR and good spatial resolution. Several filtering methods have been developed in the past decades to address denoising problem in MR images [3].

\subsection{Previous Work Done}

Denoising methods in MRI can be categorized in two groups: acquisition-based noise reduction and post acquisition-based noise reduction methods. The method for improving the signal to-noise ratio during the acquisition of an image is either increasing acquisition time or decreasing spatial resolution. However, the acquisition time is limited due to system throughput and patient comfort. Therefore, in acquisition-based method, there is a practical limit on the signal-to-noise ratio of the acquired MRI data. Hence, post acquisition image denoising is an inexpensive and effective alternative. The main objective of a post processing MRI denoising algorithm is reducing the noise power and maintaining the original resolution of the useful features in magnetic resonance images. In literature part, surveys of post acquisition-based noise reduction methods are explained [4].

Karl Krissian et al. [5] depicted Noise-Driven Anisotropic Diffusion Filtering of MRI. This method of filtering is proposed to remove the Rician noise from magnetic resonance images. It combines a Linear Minimum Mean Square Error filter and anisotropic diffusion filter (ADF).

The classic bilateral filter by Tomasi and Manduchi [6] is a weighted Gaussian filter that makes use of an intensity component in addition to the spatial component to reweight the Gaussian filter. Even though various methods for accelerating the original bilateral filter exist, it does not seem to be feasible to directly speedup the joint bilateral filtering. On the other hand, the bilateral filter is essentially a smoothing filter. It does not sharpen edges. The edge rendered by the bilateral filter has the same level of blurriness as in the original degraded image, although the noise is greatly reduced. The results of the bilateral filtering are a significant improvement over a conventional linear low-pass filter.

Non local mean algorithm takes a mean of all pixels in the image, weighted by how similar these pixels are to the target pixel. This results in much greater post-filtering clarity, and less loss of detail in the image compared with local mean algorithms [7].

All these methods introduced in the literature for removing noise from the image are computationally very complex and as image size goes on increasing, task becomes time consuming. A hardware approach to reduce the execution time of the filter is to use the multiprocessor architectures while porting the algorithm to parallel domain. With high end parallel architectures the speed up achieved is incomparably higher than the algorithmic solutions. But disadvantage of these mainframe architectures is they are costly and not programmer friendly. To make estimation of noise present in image and for removal of the same using complex algorithm is a crucial task which requires high computing capacity. In this context, hardware acceleration is crucial and Field programmable Gate Array (FPGA) best fit the growing demand of computational capabilities [8-16].

I P Skirnevskiy discuss short description of a parallel computing technology and its usage in different areas. He also provides comparison of the performance with and without using parallel computing as well as with different percentage of using CPU and parallel computing [17].

Handling the digital image processing with the help of conventional computer is accomplished only at the cost of time and conceptual distraction. Use of parallel processing for implementation of this algorithm helps us a lot [18-25].

\subsection{The Proposed Work}

The research work proposed here implements the non local mean filter using FPGA, for denoising brain MR images. The purpose of this work is to remove the Rician noise present in the MR image in much effective way in real time. FPGA used for this purpose is Spartan 6. Filter is implemented using VHDL language and ported on the kit.

\section{RESEARCH METHOD}

Consider $f$ as the noisy image and $h$ is the filtering parameter which is directly dependent on the standard deviation of noise. The estimated value of $\mathrm{i}^{\text {th }}$ pixel using NLM filter is 


$$
N L(f)(i)=\sum_{j \in I} w(i, j) f(j)
$$

where $\mathrm{w}(\mathrm{i}, \mathrm{j})$ depend on the similarity between the pixel $\mathrm{i}$ and $\mathrm{j}$ and it is between 0 to 1 and $\sum_{j} w(i, j)=1$. The similarity between the two pixel $\mathrm{i}$ and $\mathrm{j}$ depend on the similarity of intensity gray level vectors $\mathrm{f}\left(N_{i}\right)$ and $\mathrm{f}\left(N_{j}\right)$ where $N_{k}$ denotes the square neighborhood of fixed size and centered at pixel k. This similarity is measured as a decreasing function of the weighted Euclidean distance, $\|f(N i)-f(N j)\|_{2, a}^{2}$ where a>0 is the standard deviation. Pixel with similar gray level will have more weight and pixel with different gray level will have less weight. This weight $w(i, j)$ is calculated as

$$
w(i, j)=\frac{1}{z(i)} e^{-\frac{\|f(N i)-f(N j)\|_{2, a}^{2}}{h^{2}}}
$$

where $\mathrm{z}(\mathrm{i})$ is the normalizing constand and it is given by

$$
z(i)=\sum_{j} e^{-\frac{\|f(N i)-f(N j)\|_{2, a}^{2}}{h^{2}}}
$$

So for every pixel v(i) from noisy, on a defined neighborhood Nk, non local mean filter will find out the weight for every pixel depending upon the similarity between the pixel to process and the pixel in the surrounding. This filter produces very promising result compared other traditional filters but the problem associated with this filter is that this filter is computationally very intensive. So for denoising complete image of size (I, J), it takes time. So result will be avaiable after some time depending upon the size of image and the neighbourhood defined. To perform the fast compuation of the said filter, hardware is useful. So we have implemented the said algorithm on FPGA platform which increases the speed of compuation using parallel processing. This algorithm is implemented on Spartan 6 development board with device XC6SLX9 and it is compared with conventional NLM. The block diagram of proposed design is as shown in Figure 1.

Input image is applied pixel by pixel to FPGA for applying NLM algorithm. Inside the FPGA a specialized memory(SM) is designed to store the pixels received from the image. If $I$ is the input image and and estimation of $\mathrm{i}^{\text {th }}$ pixel value using $\mathrm{j}^{\text {th }}$ pixel will be as shown in Figure 2.

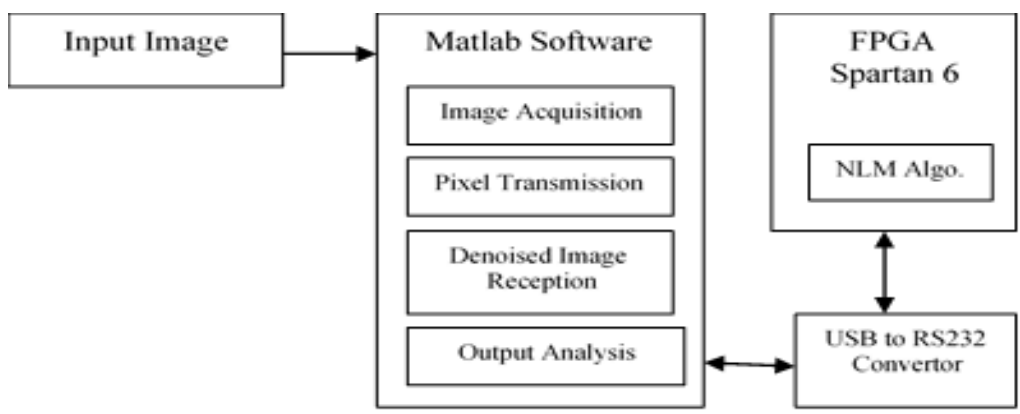

Figure 1. Block diagram of proposed design

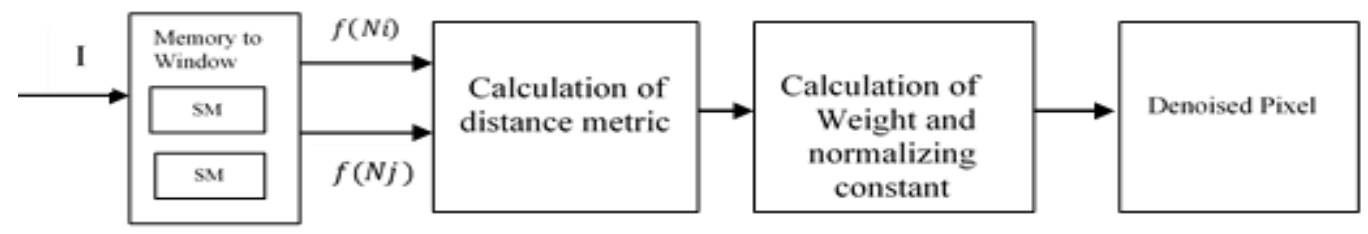

Figure 2. Flow of NLM Filter Algorithm on Spartan 6

\section{RESULTS AND DISCUSSION}

The original MR-Images are de-noised using modified NLM and NLM filter and the de-noised images are as shown in Figure 3. 

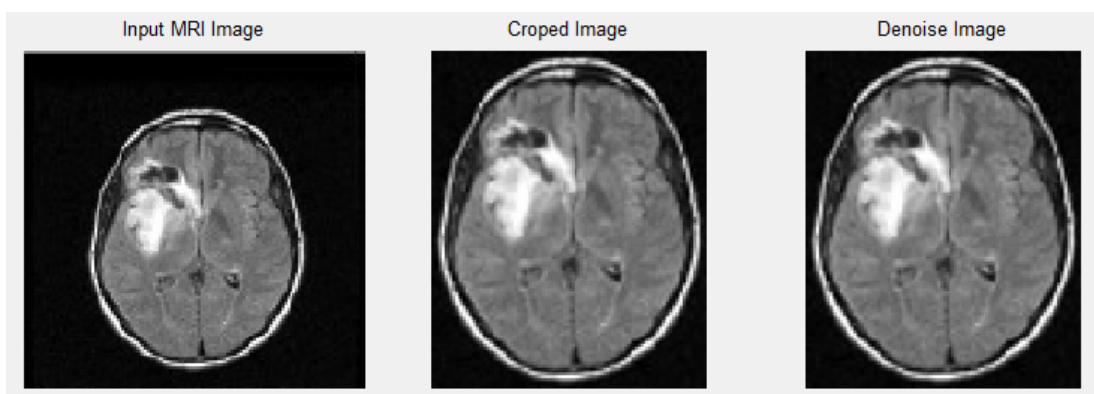

Figure 3. Original and Denoised Image Using NLM

For quantitative assessment, we have used different quality metrics such as MSE, PSNR, SSIM and PFOM. The graph of MSE, PSNR, SSIM, PFOM can be seen in Figure 4 to 8 . We have calculated MSE, PSNR, SSIM and PFOM for 50 different MRI images and plotted the calculated values w.r.t. the image. Device Utilization Summary (estimated value) of Spartan 6 is as shown in Table 1.

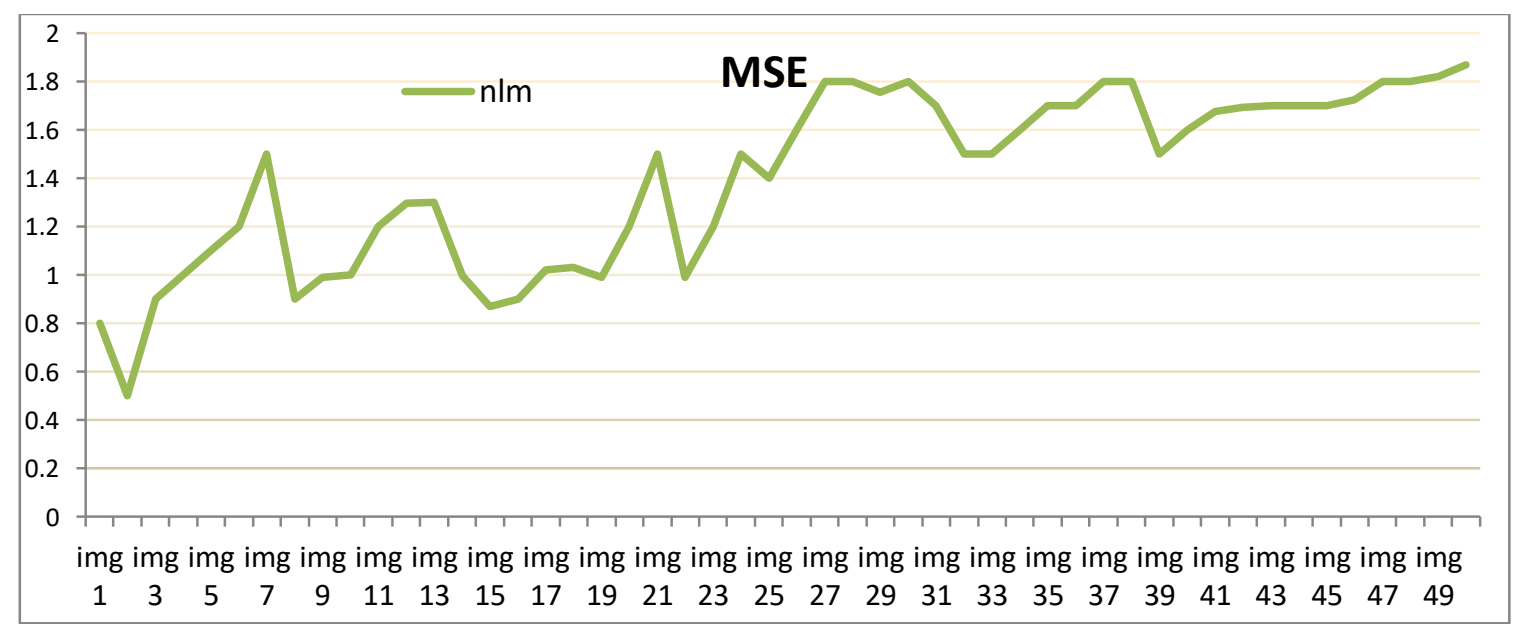

Figure 4. Calculation of MSE for 50 different images

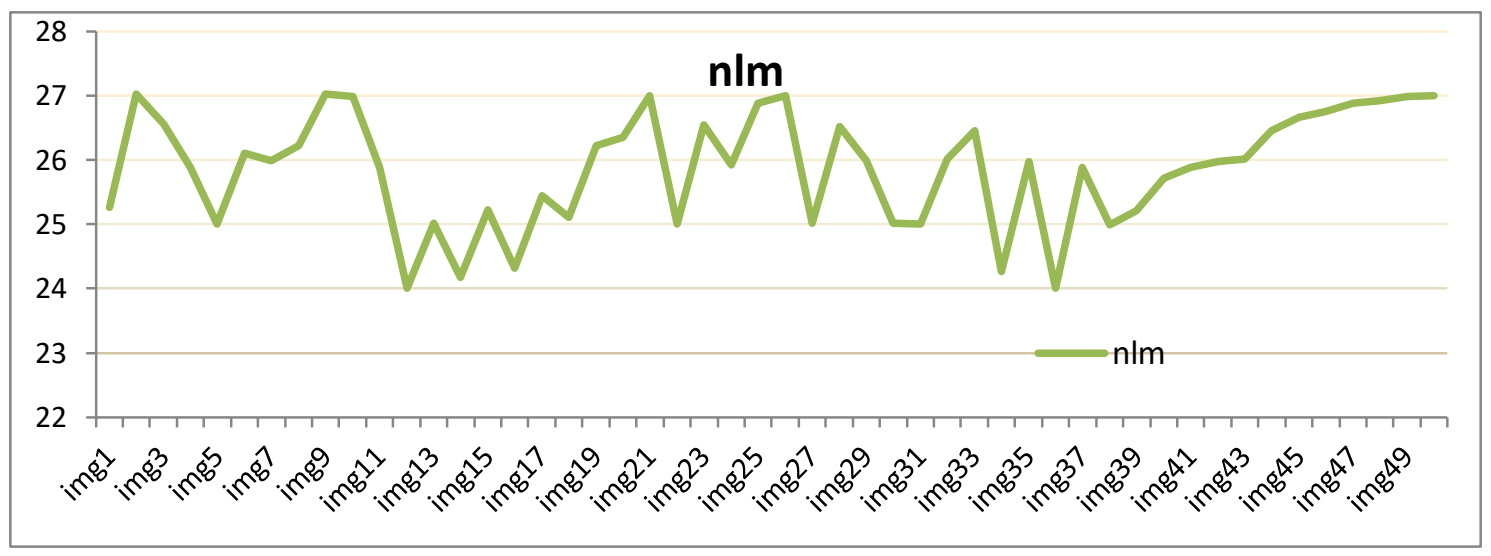

Figure 5. Calculation of PSNR for 50 different images 


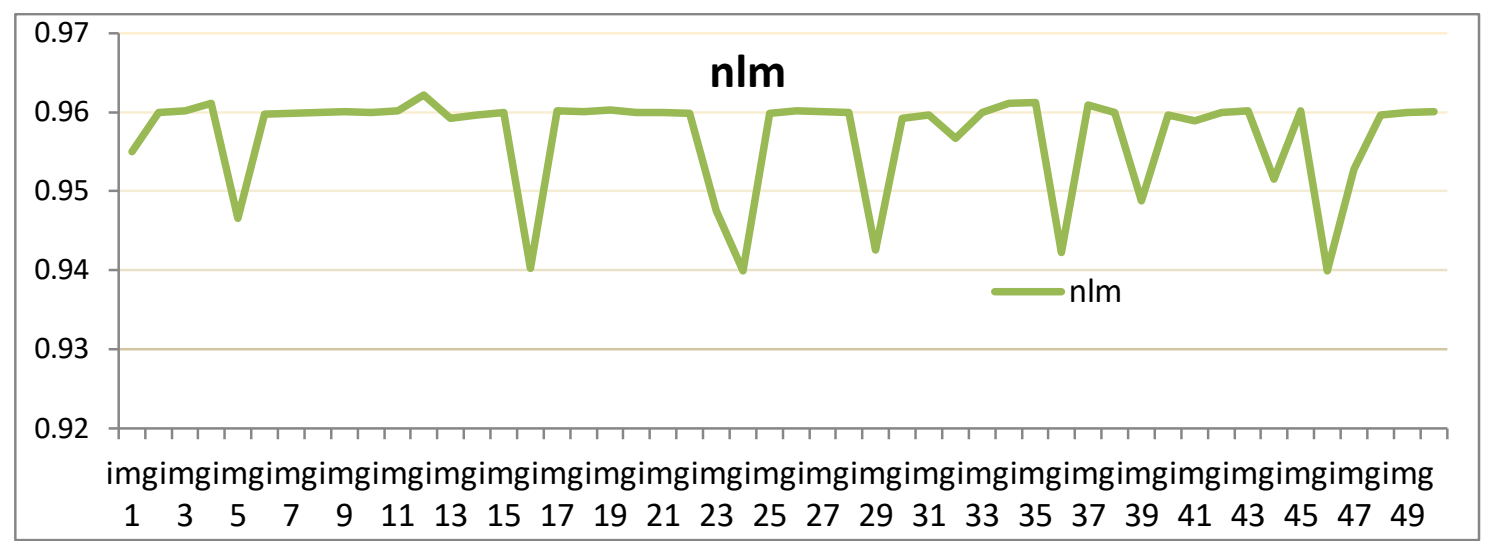

Figure 6. Calculation of SSIM for 50 different images

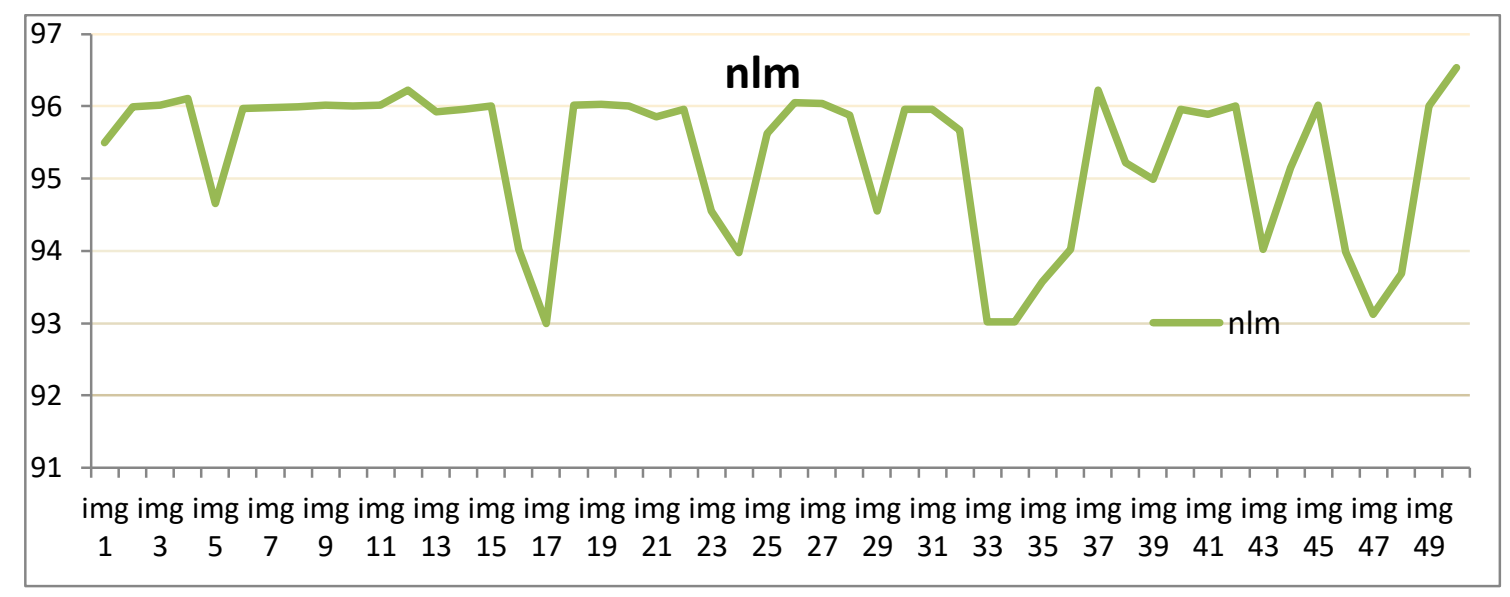

Figure 7. Calculation of PFOM for 50 different images

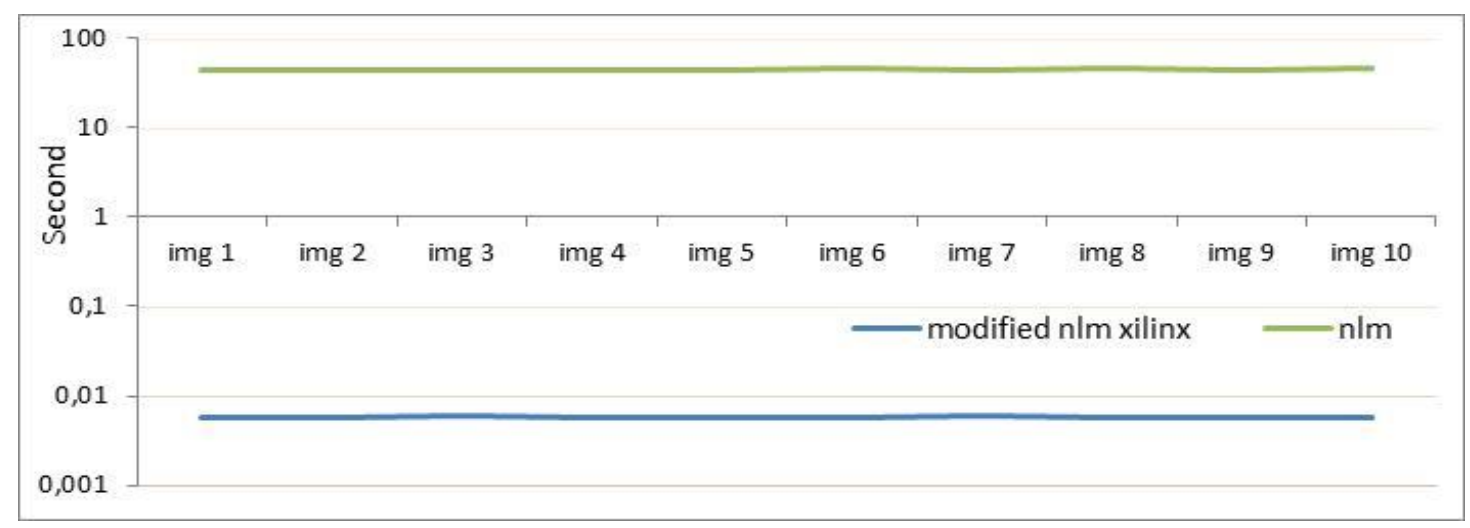

Figure 8. Processing Time on Matlab and Xilinx for 10 different images

Table 1. Device Utilization of Spartan 6

Device Utilization Summary (estimated value)

\begin{tabular}{cccc}
\hline \multicolumn{4}{c}{ Device Utilization Summary (estimated value) } \\
Logic Utilization & Used & Avaiable & Utilizaton \\
\hline Number of Slice Registers & 46 & 11440 & $0 \%$ \\
Number of Slice LUTs & 76 & 5720 & $1 \%$ \\
Number of Fully used LUT-FF pairs & 41 & 81 & $50 \%$ \\
Number of bonded IOBs & 13 & 200 & $6 \%$ \\
Number of DSP48A1s & 1 & 16 & $6 \%$ \\
\hline
\end{tabular}


From the graph of MSE, PSNR, SSIM, PFOM, we can observe that we are getting all these values near to ideal. Ideal value of SSIM has to be 1 and observed values are in the range 0.94 to 0.96 which shows very much similarity between original and denoised image. Also PFOM ideal value is $100 \%$ and the value that we have received is in the range $93 \%$ to $96 \%$. Processing time of NLM using Xilinx is very less compared to Matlab implementation.

\section{CONCLUSION}

Based on the objective quality metrics, we can conclude that filter is effectively removing noise from the MRI. We can also show that the denoised image is good in terms of structure and edge profile from SSIM and PFOM. Also SSIM and PFOM show almost 95\% matching with the original image. Also the time needed to denoise the complete image is very less compared to its matlab implementation. Algorithm implemented shows average 7000 times faster than the software implementation. So result demonstrate the advantage of implemenation of NLM using FPGA compared to Matlab, hence can be considered as useful for MRI post processing.

\section{REFERENCES}

[1] G. Wright, "Magnetic resonance imaging”, IEEE Signal Process. Mag.14, pp. 56-66, 1997.

[2] R.M. Henkelman,"Measurement of signal intensities in the presence of noise in MR images," Med. Phys. 12, pp. 232-233, 1985.

[3] Pegah Elahi, Soosan Behesht, Masoud Hashemi, "BMD MRI Denoising Equipped With Noise Invalidation Technique," 2014 IEEE International Conference on Acoustic, Speech and Signal Processing (ICASSP), pp. 6612-6616, 2014.

[4] Mohan, V. Krishnaveni, Yanhui Guo, "A survey on the magnetic resonance image denoising methods," Biomedical Signal Processing and Control, 9, pp. 56-69, 2014.

[5] K. Krissian and S. Aja-Fernandez, "Noise-driven anisotropic diffusion filtering of MRI," IEEE Transactions on Image Processing, vol. 18, no. 10, pp. 2265-2274, Oct. 2009.

[6] C. Tomasi and R. Manduchi, "Bilateral filtering for gray and color images", in the proceedings of $6^{\text {th }}$ IEEE International conference on computer vision, (1998), pp. 834-846.

[7] Buades, Antoni (20-25 June 2005). "A non-local algorithm for image denoising". Computer Vision and Pattern Recognition, 2005. 2. pp. 60-65. CiteSeerX 10.1.1.103.9157. doi:10.1109/CVPR.2005.38. ISBN 978-0-76952372-9.

[8] Fan L, Wang X, Zhang G, Chen Q., "Research about Three Dimensional Reconstruction of Medical Image," TELKOMNIKA (Telecommunication, Computing, Electronics and Control), vol. 11(2), pp. 1047-1053, 2013.

[9] Stefano Di Carlo, Paolo Prinetto, Daniele Rolfo, "AIDI:An Adaptive Image Denoising FPGA based IP Core for real time applications", IEEE, 2013, DOI: 10.1109/AHS.2013.6604232.

[10] Aqwam Rosadi Kardian, Sunny Arief Sudiro, Sarifuddin Madenda, "Efficient Implementation of Mean, Variance and Skewness Statistic Formula for Image Processing Using FPGA Device”, Bulletin of Electrical Engineering and Informatics (BEEI), Vol.7, No.3, Sep. 2018.

[11] Gian Carlo Cardarilli, Luca Di Nunzio, Rocco Fazzolari, Daniele Giardino, Marco Matta, Marco Re, Sergio Spanò, Lorenzo Simone, "Efficient FPGA implementation of high speed digital delay for wideband beamforming using parallel architectures", Bulletin of Electrical Engineering and Informatics (BEEI), Vol.8, No.2, June 2019.

[12] Takashi Saegusa, Tsutomu Maruyama and Yoshiki Yamaguchi, "How fast is an FPGA in image processing?," 2008 International Conference on Field Programmable Logic and Applications, Heidelberg, 2008, pp. 77-82. doi: 10.1109/FPL.2008.4629911.

[13] Knittel, "A PCI-compatible FPGA-coprocessor for 2D/3D image processing," 1996 Proceedings IEEE Symposium on FPGAs for Custom Computing Machines, Napa Valley, CA, USA, 1996, pp. 136-145. doi: 10.1109/FPGA.1996.564782.

[14] Zhi Guo, Walid Najjar, Frank Vahid, Kees Vissers, “A quantitative analysis of the speedup factors of FPGAs over processors", FPGA '04 Proceedings of the 2004 ACM/SIGDA 12th international symposium on Field programmable gate arrays, Pages 162-170, Monterey, California, USA-February 22 - 24, 2004, DOI: https://doi.org/10.1145/968280.968304.

[15] S. Che, J. Li, J. W. Sheaffer, K. Skadron and J. Lach, "Accelerating Compute-Intensive Applications with GPUs and FPGAs," 2008 Symposium on Application Specific Processors, Anaheim, CA, 2008, pp. 101-107. doi: 10.1109/SASP.2008.4570793.

[16] E. Nurvitadhi, D. Sheffield, Jaewoong Sim, A. Mishra, G. Venkatesh and D. Marr, "Accelerating Binarized Neural Networks: Comparison of FPGA, CPU, GPU, and ASIC," 2016 International Conference on Field-Programmable Technology (FPT), Xi'an, 2016, pp. 77-84.doi: 10.1109/FPT.2016.7929192.

[17] I P Skirnevskiy, A V Pustovit, M O Abdrashitova, "Digital image processing using parallel computing based on CUDA technology", International Conference of Technologies in Business and Industry, 2016, doi:10.1088/17426596/803/1/012152. 
[18] Sternberg S.R. (1981) "Parallel Architectures for Image Processing. In: Onoe M., Preston K., Rosenfeld A. (eds) Real-Time Parallel Computing. Springer, Boston, MA DOI : https://doi.org/10.1007/978-1-4684-3893-2_24.

[19] J. Batlle, J. Mart1, P. Ridao and J. Amat, "A New FPGA/DSP-Based Parallel Architecture for Real-Time Image Processing”, Elsevier Science Ltd, 2002, doi:10.1006/rtim.2001.027.

[20] D. Jinghong, D. Yaling and L. Kun, "Development of Image Processing System Based on DSP and FPGA," 2007 8th International Conference on Electronic Measurement and Instruments, Xi'an, 2007, pp. 2-791-2-794. doi: 10.1109/ICEMI.2007.4350799.

[21] B. A. Draper, J. R. Beveridge, A. P. W. Bohm, C. Ross and M. Chawathe, "Accelerated image processing on FPGAs," in IEEE Transactions on Image Processing, vol. 12, no. 12, pp. 1543-1551, Dec. 2003. doi: 10.1109/TIP.2003.819226.

[22] M. Fons, F. Fons and E. Canto, "Fingerprint Image Processing Acceleration Through Run-Time Reconfigurable Hardware," in IEEE Transactions on Circuits and Systems II: Express Briefs, vol. 57, no. 12, pp. 991-995, Dec. 2010. doi: 10.1109/TCSII.2010.2087970.

[23] F. Bensaali, A. Amira, A. Bouridane, "Accelerating matrix product on reconfigurable hardware for image processing applications", IEE Proceedings-Circuits, Devices and Systems, Volume 152, Issue 3, June 2005, pp. 236-246, DOI : 10.1049/ip-cds:20040838.

[24] F. M. Siddiqui, M. Russell, B. Bardak, R. Woods and K. Rafferty, "IPPro: FPGA based image processing processor," 2014 IEEE Workshop on Signal Processing Systems (SiPS), Belfast, 2014, pp. 1-6. doi: 10.1109/SiPS.2014.6986057.

[25] N. Chugh, V. Vasista, S. Purini and U. Bondhugula, "A DSL compiler for accelerating image processing pipelines on FPGAs," 2016 International Conference on Parallel Architecture and Compilation Techniques (PACT), Haifa, 2016, pp. 327-338. doi: 10.1145/2967938.2967969.

\section{BIOGRAPHIES OF AUTHORS}

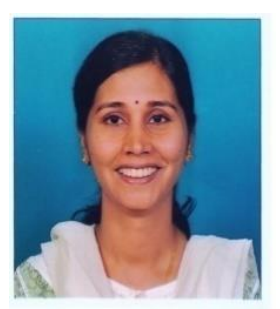

Vandana V. Hanchate has completed her bachelor's degree in Electronics and Telecommunications from Walchand Institute of Technology, Solapur, Maharashtra, India in year 1997. She completed her Masters degree in Electronics Engineering from Sinhgad College of Engineering, Maharashtra, India in year 2011. Currenlty she is pursuing her Ph. D. in Electronics and Telecommunications engineering from College of Engineering, Pune under Pune University. Having an experience of 14 years, she is currently working as a Assistant Professor in Electronics and Telecommunication Department of PES's Modern college of Engineering, Pune Maharashtra, India. Her research interests include Image processing, VLSI Design and computer vision.

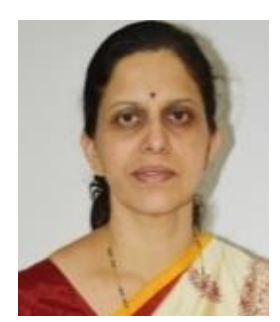

Dr. Mrs. Kalyani R. Joshi has currently been working as Principal and Professor E\&TC in PES's Modern College of Engineering, Pune, Maharashtra, India. She has completed her Bachelor's degree in Electronics Engineering from Shivaji University and Masters degree in E\&TC from College of Engineering, Pune under Pune University. She has got her Ph. D. degree from College of Engineering, Pune in year 2008 with specialization in biomedical image processing. She has been worked as Chair, Signal Processing Chapter, IEEE Pune section for three consecutive years from 2013 to 2015 and awarded as senioer member of IEEE. She has Coauthored a book on Image And Video Compression Fundamentals, CRC Press-2015. She has more than 30 national and international publications in reputed journals and conferences. Her areas of interests are Digital Signal and Image Processing and signal processing in Communication. 